\title{
Sarcoidosis complicating treatment with natalizumab for Crohn's disease
}

\author{
C A Parisinos, ${ }^{1}$ C W Lees, ${ }^{1}$ W A H Wallace, ${ }^{2}$ J Satsangi ${ }^{1}$
}

${ }^{1}$ Gastrointestinal Unit, Western General Hospital, Edinburgh, UK ${ }^{2}$ Department of Laboratory Medicine (Pathology), Edinburgh Royal Infirmary, Edinburgh, UK

\section{Correspondence to}

Professor Jack Satsangi, Gastrointestinal Unit, Molecular Medicine Centre, University of Edinburgh, Western General Hospital, Crewe Road, Edinburgh EH4 2XU, UK; j.satsangi@ed.ac.uk

Received 16 November 2010 Accepted 9 December 2010 Published Online First 13 January 2011

\section{ABSTRACT}

Natalizumab is a humanised monoclonal antibody targeting the lymphocyte adhesion molecule a4 integrin, with proven efficacy in multiple sclerosis (MS) and Crohn's disease (CD). The development of sarcoidosis with extrapulmonary involvement is reported in two patients with refractory CD who had received maintenance therapy with natalizumab. This complication has not been previously reported. It is hypothesised that the effect of natalizumab in altering lymphocyte mucosal trafficking may underlie the development of sarcoidosis in these patients.

\section{CASE REPORTS \\ Case 1}

A 38-year-old man with Crohn's disease (CD) was referred into a phase II clinical trial of natalizumab. His past medical history included 20 years of colonic CD and pancreatitis secondary to azathioprine. Following initial infusions of natalizumab (6 mg/kg) in December 2002 and January 2003, the patient entered the maintenance arm $(300 \mathrm{mg}$ natalizumab monthly).

During the study the patient developed a dry cough. A chest x-ray in May 2004 revealed peripheral infiltrates and hilar lymphadenopathy; carbon monoxide transfer factor (TCO) was $73 \%$ predicted and serum ACE was increased (95 U/1). A highresolution CT (HRCT) scan of the thorax showed lung nodules with left lower lobe conglomeration. A transbronchial biopsy demonstrated several epithelioid non-caseating granulomas consistent with sarcoidosis (figure 1C). Natalizumab was withdrawn (total nine infusions) and systemic corticosteroids started (complicated by psychosis; completed July 2005). One year later he developed increased dyspnoea. The chest $\mathrm{x}$-ray (figure 1A) and HRCT scan (figure 1B) demonstrated extensive pulmonary sarcoidosis. Treatment was started with methotrexate and subsequently adalimumab. In January 2009 the patient presented with a truncal papular rash. Biopsy revealed granulomatous dermatitis. Remission has since been maintained with methotrexate $5 \mathrm{mg}$ and adalimumab $40 \mathrm{mg}$ once weekly.

\section{Case 2}

A 37-year-old man with CD was referred into the phase II clinical trial of natalizumab. His past medical history included 22 years of $\mathrm{CD}$ with ileocaecal resection (1992; postoperative azathioprine). The patient had 17 monthly $300 \mathrm{mg}$ doses of natalizumab. On subsequent questioning the patient had noted a dry cough towards the end of this treatment phase in 2005.

After the trial the patient was managed with azathioprine, which was discontinued by September 2008 after prolonged remission. In December 2008 the patient's cough became worse and was accompanied by a red left eye, myalgia and a rash on both shins. The chest x-ray showed clear evidence of bilateral hilar lymphadenopathy (figure 1D); Tco was $82 \%$ predicted, and the HRCT scan demonstrated mediastinal and hilar lymphadenopathy (figure 1E). A mediastinoscopy was performed, with histological appearances demonstrating non-necrotising granulomatous lymphadenitis, consistent with sarcoidosis (figure $1 \mathrm{~F}$ ). We hypothesised that azathioprine therapy following termination of the trial had initially controlled the disease. The patient remained on high-dose steroid therapy (20 $\mathrm{mg}$ prednisolone) until September 2009 due to refractory sarcoidosis.

\section{DISCUSSION}

Sarcoidosis and CD are inflammatory barrier disorders with common features including nonnecrotising granulomas, dysregulated $T$ cell activation and similar organ involvement outside the lung and gut. A genome-wide analysis in sarcoidosis and CD detected a common susceptibility locus on 10p12.2. ${ }^{1}$ Moreover, polymorphisms in the CD susceptibility gene NOD2 have been implicated in early-onset pulmonary sarcoidosis. ${ }^{2}$

Natalizumab inhibits the binding of $\alpha 4$ integrin to vascular cell adhesion molecule- 1 and mucosal addressin cell adhesion molecule-1, a critical step in the migration of lymphocytes into the CNS and gut. $^{3}$ We hypothesise that this may contribute to the development of sarcoidosis by preventing trafficking of proinflammatory lymphocytes to the gut, allowing dysregulated traffic to the respiratory mucosa and other extraintestinal mucosal surfaces.

Natalizumab has achieved FDA approval for the treatment of refractory $\mathrm{CD}$ and multiple sclerosis. However, it has received very close assessment following reports of progressive multifocal leucoencephalopathy complicating its use. ${ }^{4}$ This report highlights the need for vigilance in the use of biological agents in immune-mediated diseases. 
Figure 1 Case 1. (A) Chest x-ray in 2006 showing new widespread nodular opacification, suspicious of a progressive interstitial process. (B) High-resolution CT scan in 2006 showing extensive bilateral symmetrical fine pulmonary nodularity involving all lobes of the lung. (C) Histological section taken from a transbronchial biopsy specimen in 2004 showing the presence of epithelioid non-caseating granulomas consistent with sarcoidosis. Stains for fungus (DPAS and Grocott) and acidfast bacilli (ZN) were negative $(\mathrm{H \& E}, \times 200)$. Case 2. (D) Chest $x$-ray in 2008 showing bilateral hilar lymphadenopathy. (E) High-resolution CT scan in 2009 showing prominent hilar and mediastinal lymph nodes (arrow). (F) Histological section from a mediastinoscopy in 2009 showing non-necrotising granulomas (arrows) replacing the predominant part of the lymph node tissue, consistent with sarcoidosis. DPAS, Grocott and ZN stains were negative $(\mathrm{H \& E}, \times 25)$.
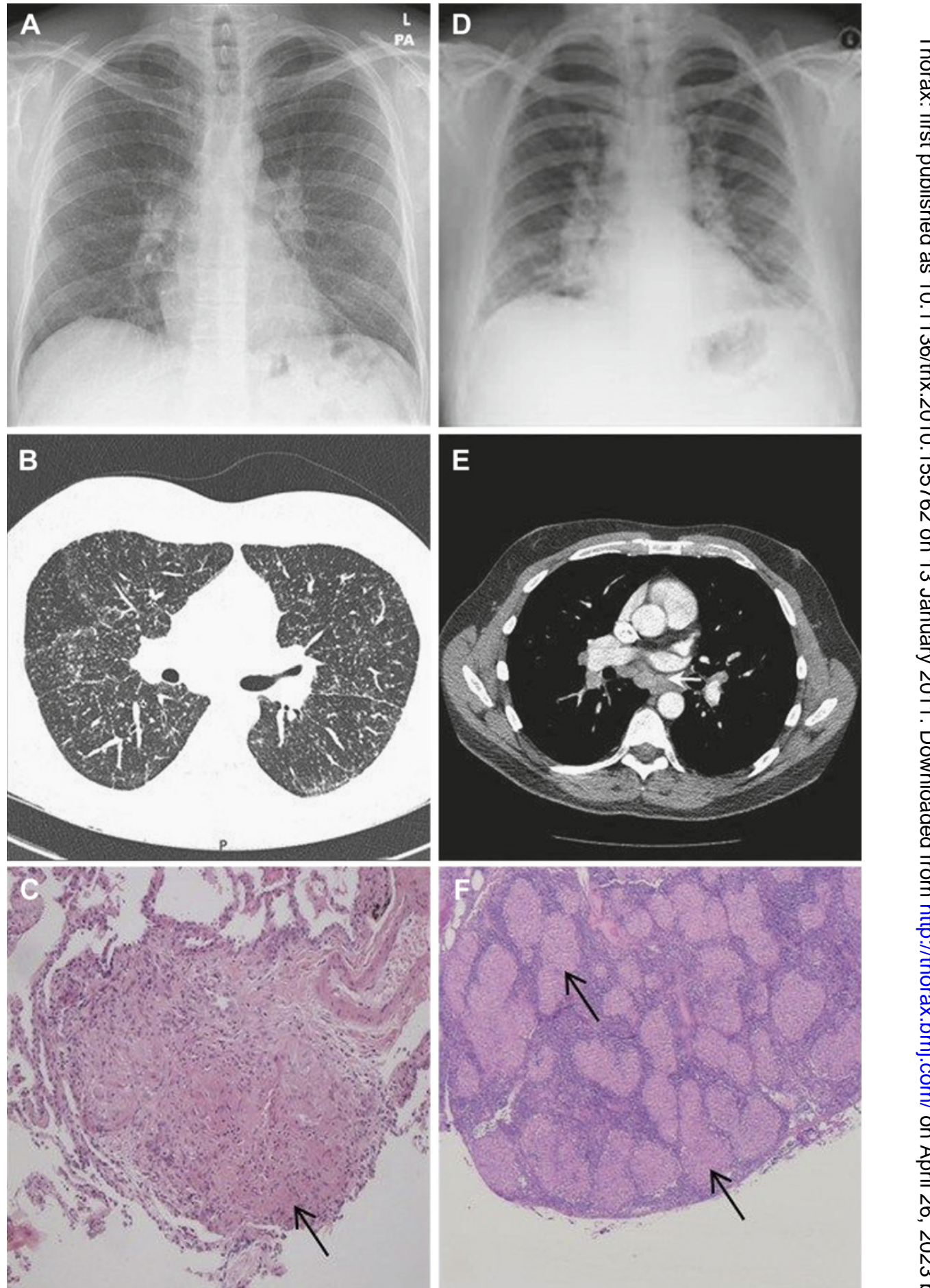

Acknowledgements The authors would like to thank Dr Helen Rodgers and Dr Joaquim Majo for their helpful input.

Competing interests CWL has a consultancy with Abbott, payment for lectures by Abbott, MSD, Ferring and Shire and a patent pending with Genentech. JS declares one paid consultancy (Elan Biotech) and financial support for the natalizumab trial from Elan, grants with Novartis and Genentech and research funding by Schering Plough. CAP and WAHW have no conflicts of interest.

Patient consent Obtained.

Contributors CAP: literature search, writing, figures, editing. CWL: writing, editing. WAHW: pathology images. JS: initial hypothesis, main editor of manuscript and writing.
Provenance and peer review Not commissioned; internally peer reviewed.

\section{REFERENCES}

1. Franke A, Fischer $A$, Nothnagel $M$, et al. Genome wide association analysis in sarcoidosis and Crohn's disease unravels a common susceptibility locus on 10p12.2. Gastroenterology 2008;135:1207-15.

2. Sakai $\mathbf{H}$, Ito $S$, Nishikomori R, et al. A case of early onset sarcoidosis with a six base deletion in the NOD 2 gene. Rheumatology 2010:62:250-7.

3. von Andrian UH, Engelhardt B. Alpha4 integrins as therapeutic targets in autoimmune disease. N Engl J Med 2003;348:68-72.

4. Adelman B, Sandrock A, Panzara MA. Natalizumab and progressive multifocal leukoencephalopathy. N Engl J Med 2005;353:432-3. 